\title{
Comparative evaluation of intranasally delivered quetiapine loaded mucoadhesive microemulsion and polymeric nanoparticles for brain targeting: pharmacokinetic and gamma scintigraphy studies
}

\author{
Brijesh Shah ${ }^{1,2^{*}}$ (D), Dignesh Khunt ${ }^{3}$ (D) and Manju Misra ${ }^{1}$ (D)
}

\begin{abstract}
Background: Treatment in neurological disorders like schizophrenia requires continuous presence of drug in the brain for a prolonged period of time to achieve an effective therapeutic response. Delivery of antipsychotic drug quetiapine in the form of conventional delivery systems suffers from low oral bioavailability, first-pass metabolism, and frequent dosing. In addition to that biological obstacles present at the brain interface also hinders the transport of quetiapine across the brain. In the present study, nasal delivery of quetiapine loaded nanoparticles and microemulsion formulation were designed to evaluate their individual in vivo potential to achieve brain targeting. Chitosan-based polymeric nanoparticles and mucoadhesive microemulsion systems were developed through ionic gelation and water titration method respectively.

Results: Microemulsion showed globule size lower than $50 \mathrm{~nm}$ with 95\% drug loading while, nanoparticles exhibited 65\% drug loading with particle size of $131 \mathrm{~nm}$. Nasal diffusion study showed highest diffusion with chitosan-based mucoadhesive microemulsion over nanoparticles suggesting permeation-enhancing effects of chitosan. Due to the overall hydrophilic nature, quetiapine-loaded nanoparticles could not diffuse superiorly across nasal mucosa, hence, showed 1.3 times lesser diffusion compared to mucoadhesive microemulsion. Pharmacokinetics in rats showed highest brain concentration and 1.9-folds higher nasal bioavailability with mucoadhesive microemulsion over nanoparticles suggesting direct brain transport through olfactory route bypassing blood-brain barrier.

\footnotetext{
* Correspondence: brijeshshah25@ymail.com

'Department of Pharmaceutics, B. V. Patel PERD Centre, Ahmedabad 380054, India

${ }^{2}$ Department of Pharmaceutical Sciences, College of Pharmacy, University of

North Texas Health Science Center, Fort Worth, TX 76107, USA

Full list of author information is available at the end of the article
}

\section{Springer Open}

() The Author(s). 2021 Open Access This article is licensed under a Creative Commons Attribution 4.0 International License, which permits use, sharing, adaptation, distribution and reproduction in any medium or format, as long as you give appropriate credit to the original author(s) and the source, provide a link to the Creative Commons licence, and indicate if changes were made. The images or other third party material in this article are included in the article's Creative Commons licence, unless indicated otherwise in a credit line to the material. If material is not included in the article's Creative Commons licence and your intended use is not permitted by statutory regulation or exceeds the permitted use, you will need to obtain permission directly from the copyright holder. To view a copy of this licence, visit http://creativecommons.org/licenses/by/4.0/. 


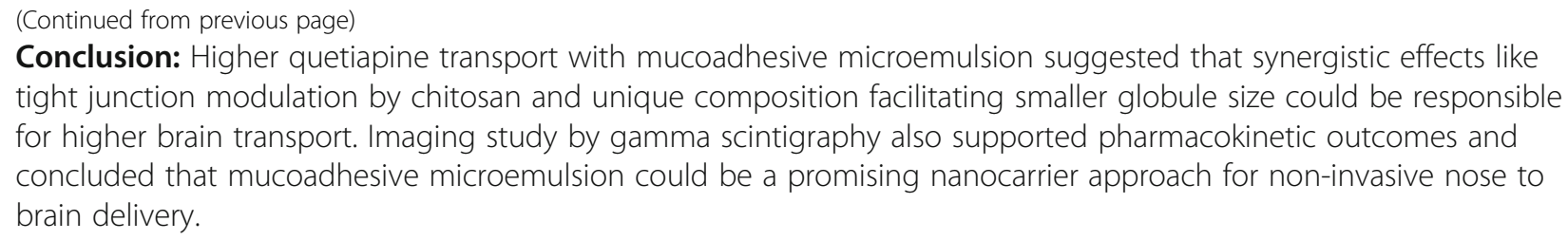

Keywords: Brain targeting, Intranasal delivery, Quetiapine, Polymeric nanoparticles, Mucoadhesive microemulsion, Gamma scintigraphy

\section{Background}

Despite extensive advancements in targeting technologies, brain targeting is still considered challenging by formulation scientists, when it comes to drug delivery in the area of neurological disorders. Treatment of central nervous system (CNS) disorders is significantly hampered by the complex physiology of the brain, accounting for more than 7 million global deaths every year [1]. The brain is surrounded by biological obstacles such as blood-brain barrier (BBB) and efflux transporter proteins whereby tight junctions (TJ) and P-glycoprotein (Pgp) act as specialized interface between the systemic circulation, i.e., blood and brain parenchyma [2]. These obstacles behave as gatekeepers, hence, systemic delivery of majority of neurotherapeutics in the form of conventional oral dosage form is rendered ineffective, as the desired concentration is not achieved in the brain. In addition to that, therapeutics given via oral route have to face a challenging gastrointestinal environment and hepatic-first pass metabolism, followed by crossing BBB, drugs reach to the brain. As a result, conventional drug delivery is often marred with lower concentration, poor efficacy, side-effects associated with systemic exposure, and frequent dosing. To overcome these limitations, drug may be delivered directly to the brain by overcoming and/or disrupting BBB.

Drug delivery strategies across the BBB comprises of either invasive methods involving risky surgical interventions or patient-friendly non-invasive approaches like chemical or biological modifications and intranasal delivery. Invasive methods like BBB disruption, intracerebral implants, or intraventricular infusion involve irreversible disruption of barrier integrity, often resulting into severe neurological changes, alteration of brain functions, and brain abscess on prolonged time [3]. In contrast, non-invasive intranasal (IN) route avoids surgical procedures and delivers drugs directly to the brain through the olfactory or trigeminal pathway by overcoming the $\mathrm{BBB}$. To some extent, IN route also delivers drug systemically crossing BBB. Rich vasculature, avoidance of first-pass metabolism, rapid onset of action, and very less systemic exposure are key advantages, making IN route promising over other delivery routes [4].
Numerous distinguished groups have published several informative reviews and researches with strong evidences and outcomes in the last three decades, which had shown a great potential of nose to brain targeting for the treatment of various CNS disorders [5-11].

Schizophrenia is one such chronic, severe, and most disabling psychiatric brain disorder, characterized by positive, negative, and cognitive symptoms, which upon long term significantly affect brain structure and functions [12]. Typical and atypical antipsychotics are choices of medications in controlling psychotic symptoms and for behavioral impairment. However, atypical antipsychotics are even more efficient over typical ones, due to their improved effects on hallucinations, delusions, and have a lower risk of extrapyramidal symptoms [13]. Among widely used atypical drugs, viz., olanzapine, quetiapine, and risperidone, quetiapine is reported to have superior therapeutic efficacy and cognitive properties [14]. However, it has few deprived properties like poor oral bioavailability $(9 \%)$, poor plasma half-life $(6 \mathrm{~h})$, extensive metabolism, requiring frequent oral dosing, and poor water solubility, besides being Pgp substrate candidate. All these challenges often results into ineffective oral treatment; however, they can be easily surmounted via IN delivery.

With the increasing demand of site-specific therapy, colloidal carriers ranging in size from $10-400 \mathrm{~nm}$ are being explored tremendously for targeting to the various sites of the body. Nanocarriers like polymeric and, lipidic nanoparticles, liposomes, nano and microemulsions are believed to protect the drugs via encapsulation, offer high payload, and their smaller particle size allows easy access across biological membranes like BBB, thereby producing higher therapeutic responses even at low doses. In the past few years, extensive experiments have been performed on the nose to brain targeting using polymeric and lipidic carriers systems, wherein few groups have outlined the superiority of lipidic systems over polymeric carriers in achieving higher brain targeting $[15,16]$. However, we could hardly come across any work wherein two different carrier systems have been explored for one drug and compared in terms of pharmacokinetics and brain distribution imaging studies. 
The present investigation is aimed at developing polymeric nanoparticles (NP) and mucoadhesive microemulsion (MME) formulations of Pgp substrate molecule, quetiapine fumarate. Quetiapine-loaded NP and MME were developed through ionic gelation and water titration method respectively and characterized for size, morphology, ex vivo nasal diffusion, pharmacokinetic evaluation, and qualitative imaging analysis to determine brain targeting potential and biodistribution of quetiapine through individual formulations.

\section{Materials}

Quetiapine fumarate was a kind gift sample from Torrent Pharmaceuticals Ltd. (Ahmedabad, India). Capmul MCM-EP was provided as gift samples from Abitec Corporation Ltd. (Mumbai, India). Labrasol and Transcutol-P were gift samples received from Gattefosse Pvt. Ltd. (Mumbai, India). Tween-80, low molecular weight chitosan $(\mathrm{CH})$, and sodium tripolyphosphate (TPP) were purchased from Sigma-Aldrich (Bangalore, India). All other chemicals and reagents were of highly purified grade and were used without further purification.

\section{Methods}

\section{Preparation of nanoparticles}

Quetiapine-loaded nanoparticles (QNP) were prepared by ionic gelation method, which involves interaction between cationic $\mathrm{CH}$ and anionic TPP. $\mathrm{CH}$ and TPP were dissolved separately into purified water $(\mathrm{CH}$ was dissolved at $\mathrm{pH} 4.5$ ) and filtered to obtain a clear solution. Process parameters like polymer concentration, volume, stirring time, and speed were optimized as a preliminary screening to obtain desired quality attributes such as narrow size, lower polydispersity index (PDI), and higher encapsulation. Preliminary optimization revealed that concentration of $\mathrm{CH}$ and TPP, and volume of TPP had a major effect on quality attributes while stirring speed and time had less effect and hence they were set as fixed parameters. Detailed optimization through Box-Behnken design, model validation, and impact of process parameters on quality attributes, etc. have been published by our group [17]; hence, only optimized procedure is discussed in this article. Stirring time (15 min), speed (700 $\mathrm{rpm}$ ) and amount of quetiapine (1\% w/v of total volume) were kept constant, while optimized $\mathrm{CH}$ and TPP concentration, TPP volume, and $\mathrm{CH} / \mathrm{TPP}$ mass ratio were $0.1 \% \mathrm{w} / \mathrm{v}, 2.08 \mathrm{ml}$ and 4.8:1 respectively. Briefly, QNP were formulated by dissolving quetiapine completely into $\mathrm{CH}$ solution, into which TPP solution was added dropwise and allowed to stir on a magnetic stirrer to form NP dispersion. This dispersion was centrifuged at $15,000 \mathrm{rpm}$ for $30 \mathrm{~min}$ to obtain NP pellet, which was subsequently washed with purified water and redispersed into $5 \% \mathrm{w} / \mathrm{v}$ mannitol solution to obtain freeze-dried QNP. Composition of QNP is described in Table 1.

\section{Preparation of microemulsion}

Quetiapine-loaded ME (QME) was prepared by titration method. Solubility of quetiapine was performed in different oils, surfactants, and cosurfactants to determine into which vehicle the maximum amount of quetiapine is soluble. Taking these vehicles, further pseudo-ternary plots were constructed against distilled water by spontaneous emulsification method to evaluate at which surfactant: cosurfactant ratio $\left(S_{\text {mix }}\right)$, maximum ME formation takes place. The Triplot software (Ver: 4.12) was used for plotting the phase diagrams. Detailed methods of solubility study and ternary plots were published in our previous report [18], where Capmul MCM (oil), Tween 80 and, Labrasol (surfactants), and Transcutol-P (cosurfactant) showed maximum solubility for quetiapine. Briefly, quetiapine $(50 \mathrm{mg})$ was allowed to solubilize into Capmul MCM $(0.3 \mathrm{ml})$, and then $S_{\text {mix }}(2.2 \mathrm{ml})$ was added and allowed to mix until the drug is solubilized completely. Predetermined amount of distilled water $(2.5 \mathrm{ml})$ was added dropwise into the above oil and $S_{\text {mix }}$ mixture and allowed to stir until a transparent mixture of o/w QME is formed. $\mathrm{CH}$-based mucoadhesive $\mathrm{ME}$ of quetiapine (QMME) was also developed similarly by dissolving $\mathrm{CH}$ into distilled water in a manner that final QMME contains $0.5 \% \mathrm{w} / \mathrm{v}$ of $\mathrm{CH}$. Optimized formula for QME and QMME is depicted in Table 2.

\section{Characterizations of the developed formulations}

Physicochemical parameters such as size, PDI, zeta potential, drug loading capacity, $\mathrm{pH}$, and viscosity were measured for QNP, QME, and QMME. Individual formulations $(1 \mathrm{ml})$ were taken into disposable polystyrene cuvettes to measure size, PDI, and zeta potential by photon correlation spectroscopy using Zetasizer (NanoZS90, Malvern, Worcestershire, UK). Drug loading was performed by taking individual formulations equivalent to $2 \mathrm{mg}$ of quetiapine into methanol and after suitable dilutions, HPLC analysis was performed to determine loading capacity. $\mathrm{pH}$ measurements were performed at room temperature by taking $10 \mathrm{ml}$ of formulations in a small beaker. Viscosity was determined at room temperature, $30 \mathrm{rpm}$, and spindle S18 using Brookfield viscometer DV- $\mathrm{II}^{+}$(Brookfield Engineering Laboratories, Middleborough, MA). All characterizations were performed in triplicate.

\section{Morphological characterization}

The morphology of QNP, QME, and QMME was performed using TEM (Philips, Tecnai 20, Holland) at an acceleration voltage of $200 \mathrm{kV}$ and viewed at a magnification of $\times 50,000$. After suitable dilutions with distilled 
Table 1 Composition and physicochemical characterization of quetiapine loaded nanoparticles (QNP) (data represents mean \pm SD, $n=$ 3)

\begin{tabular}{lllllllll}
\hline Formulations & $\begin{array}{l}\text { CH:TPP } \\
\text { mass ratio }^{\mathbf{a}}\end{array}$ & $\begin{array}{l}\text { Drug } \\
(\% \mathbf{w} / \mathbf{v})\end{array}$ & $\begin{array}{l}\text { Drug loading } \\
(\%)\end{array}$ & Size $(\mathbf{n m})$ & PDI & $\begin{array}{l}\text { Zeta potential } \\
(\mathbf{m V})\end{array}$ & pH & Viscosity (cP) \\
\hline QNP & $4.8: 1$ & 1 & $64.29 \pm 2.12$ & $131.08 \pm 7.45$ & $0.252 \pm 0.064$ & $34.4 \pm 1.87$ & $5.87 \pm 0.11$ & $7.54 \pm 0.22$ \\
\hline
\end{tabular}

${ }^{a}$ Concentration of $\mathrm{CH}$ and TPP remains $0.1 \% \mathrm{w} / \mathrm{v}$

water, individual samples were placed on a carboncoated copper grid and stained with $1 \%$ aqueous solution of phosphotungstic acid and allowed to dry. After drying, images were captured and globules were interpreted in terms of size using the AnalySIS ${ }^{\circ}$ software (Soft Imaging Systems, Reutlingen, Germany).

\section{Nasal diffusion study}

Comparative ex vivo nasal diffusion study was performed in triplicate between QNP, QME, QMME, and drug solution (DS) using Franz diffusion cell method (Hanson Research-Telemodul 40S, Chatsworth, CA). Piece of freshly excised goat nasal mucosa was cleaned thoroughly using phosphate buffer saline (PBS) $\mathrm{pH} 6.4$ to remove any adhered tissues from nasal mucosa and further it was utilized as a dialyzing membrane. Diffusion cell with a receptor volume capacity of $12.5 \mathrm{ml}$ was taken, onto which nasal mucosa was mounted in a way so that mucosal surface faces donor compartment and serosal surface faces receptor chamber. Receptor chamber was filled with PBS pH 6.4 and kept under stirring. Temperature of the PBS was controlled at $37 \pm 2^{\circ} \mathrm{C}$ with a circulating water bath and was gassed with splash of oxygen through the aerator to ensure minimum living condition of the mucosa. Donor compartments were placed on top of the receptor chambers positioned with nasal mucosa and fixed with clamps. Liquid formulations equivalent to $10 \mathrm{mg}$ of quetiapine were placed in the donor chamber and diffusion was allowed to run for 12 h. Aliquots of $1 \mathrm{ml}$ were withdrawn at different time intervals $(0.5,1,2,4,6,8$, and $12 \mathrm{~h})$, replaced with an equal volume of PBS, and analyzed by HPLC to evaluate the percentage drug diffused [18].

\section{Pharmacokinetic study}

Animals were provided by the Department of Pharmacology and Toxicology, B.V. Patel PERD Centre, Gujarat,
India. Animal experiments and study protocols were approved by the Institutional Animal Ethics Committee of B.V. Patel PERD Centre, Gujarat, India (Registration No: 1661/PO/A/12/CPCSEA) under CPCSEA, Delhi, India.

Sprague Dawley rats weighing between $250-300 \mathrm{~g}$ were selected for the biodistribution study. Animals were divided into six groups, each consisting of 20 animals. The first four groups received QNP, QME, QMME, and DS via nasal route, while the fifth and sixth groups were given QNP and QME intravenously (IV) equivalent to $2.3 \mathrm{mg} / \mathrm{kg}$ dose of quetiapine respectively. For IN administration groups, formulation volume equivalent to 0.3 mg of quetiapine was administered into each nostril of anesthetized rats. During IN dosing, rats were held from back in slanted position, while IV dosing was given through rat tail vein and formulation volume equivalent to $0.6 \mathrm{mg}$ of quetiapine was injected. Blood samples were collected from retro-orbital vein into heparinized tubes at different time points, viz., 15, 30, 60, 120, and $240 \mathrm{~min}$ ( $n=4 /$ time point). Following blood collection, brain tissues were collected by sacrificing animals through the cervical dislocation method. Brain tissue was collected in a tube containing PBS pH 6.4 solution. Plasma was collected from blood samples through centrifugation at $8000 \mathrm{rpm}$ for $10 \mathrm{~min}$ at $4{ }^{\circ} \mathrm{C}$. Similarly, PBS pH 6.4 containing brain tissues were homogenized at $10000 \mathrm{rpm}$ and then centrifuged as mentioned for blood samples to collect supernatant. Quetiapine was extracted from plasma and brain tissue and analyzed through HPLC as per detailed bioanalytical procedure described in our previous report [18].

Pharmacokinetics profile of quetiapine in brain and plasma after IN and IV administration were determined to calculate pharmacokinetic parameters like $C_{\max }, T_{\max }$, AUC $_{0-240}$, and $T_{1 / 2}$, using pharmacokinetic software (PK Functions for Microsoft Excel, Pharsight Corporation, Mountain View, CA). Brain targeting efficiency, direct

Table 2 Composition and physicochemical characterization of quetiapine loaded microemulsion (QME) and mucoadhesive microemulsion (QMME) (data represents mean $\pm \mathrm{SD}, n=3$ )

\begin{tabular}{llllllllll}
\hline Formulations* & Oil & $\begin{array}{l}\boldsymbol{S}_{\text {mix }}{ }^{\mathbf{a}} \\
(\% \mathbf{w} / \mathbf{w})\end{array}$ & Water & $\begin{array}{l}\text { Drug loading } \\
(\mathbf{\%})\end{array}$ & $\begin{array}{l}\text { Globule size } \\
(\mathbf{n m})\end{array}$ & PDI & $\begin{array}{l}\text { Zeta potential } \\
(\mathbf{m V})\end{array}$ & pH & Viscosity (cP) \\
\hline QME & 6 & 44 & 50 & $96.18 \pm 1.23$ & $29.75 \pm 0.99$ & $0.221 \pm 0.01$ & $2.77 \pm 0.51$ & $5.94 \pm 0.08$ & $17.5 \pm 0.69$ \\
QMME $^{b}$ & 6 & 44 & 50 & $95.43 \pm 0.92$ & $35.31 \pm 1.71$ & $0.249 \pm 0.03$ & $20.29 \pm 1.23$ & $4.98 \pm 0.12$ & $38.5 \pm 1.26$ \\
\hline
\end{tabular}

${ }^{*}$ Concentration of quetiapine fumarate in each formulation is $23 \mathrm{mg} / \mathrm{ml}$

${ }^{a} S_{\text {mix }}$ ratio for Labrasol+Tween 80 : Transcutol-P is $3(1: 1): 1$

${ }^{\mathrm{b}} \mathrm{Chitosan}$ concentration in QMME is $0.5 \% \mathrm{w} / \mathrm{v}$ 
transport percentage, and nasal bioavailability were also determined through the following formulas [17]. Drugtargeting efficiency (DTE \%) represents time average partitioning ratio.

$$
\% D T E=\frac{\left(A U C_{\text {brain }} / A U C_{\text {blood }}\right) I N}{\left(A U C_{\text {brain }} / A U C_{\text {blood }}\right) I V} \times 100
$$

Direct transport percentage (DTP \%) explains nose to brain direct transport.

$$
\% D T P=\frac{B_{I N}-B_{X}}{B_{I N}} \times 100
$$

where $B_{X}=\left(\mathrm{B}_{\mathrm{IV}} / \mathrm{P}_{\mathrm{IV}}\right) \times P_{I N}$

Here, $B_{X}$ is the brain AUC fraction contributed by systemic circulation through BBB following IN administration. $B_{I V}$ is the $\mathrm{AUC}_{0-240}$ (brain) following IV administration. $P_{I V}$ is the $\mathrm{AUC}_{0-240}$ (blood) following IV administration. $B_{I N}$ is the $\mathrm{AUC}_{0-240}$ (brain) following IN administration. $\mathrm{P}_{I N}$ is the $\mathrm{AUC}_{0-240}$ (blood) following IN administration.

Nasal bioavailability was calculated as the ratio of $\mathrm{AUC}_{\mathrm{IN}} / \mathrm{AUC}_{\mathrm{IV}}$.

\section{In vivo biodistribution study using gamma scintigraphy}

To determine the in vivo fate of biodistribution after IN administration of formulation, imaging analysis is one of the most reliable and accurate tools. Gamma scintigraphy study is one such non-invasive imaging analysis technique. It was performed after approval of protocol by the Institutional Animal Ethics Committee as discussed in the previous section.

Formulations (QNP, QME, QMME, and DS) were labeled with technetium-99 $\mathrm{m}\left({ }^{99 \mathrm{~m}} \mathrm{Tc}\right)$ by direct labeling method. Individual formulations were taken in a vial and incubated for $10 \mathrm{~min}$ in the presence of stannous chloride, sodium bicarbonate, and pertechnetate $(5 \mathrm{mCi})$. After incubation, the final volume was adjusted with saline solution to obtain a radioactivity of $100 \mu \mathrm{Ci} / 20 \mu \mathrm{l}$ formulation. Radiolabeling efficiency of the formulation was confirmed through thin layer chromatography (TLC) using silica gel-coated fiber sheets against TLC strips with plain pertechnetate solution as a control. Gamma scintillation counter was used to measure the radioactivity or \% radiolabeling efficiency [19].

Sprague Dawley rats weighing between 250 and $300 \mathrm{~g}$ were selected for the gamma scintigraphy study. As discussed in the pharmacokinetic study, animals were divided into six groups, whereby $20 \mu$ of radiolabeled drug formulations were administered to anesthetized rats via IN (QNP, QME, QMME, and DS) and IV (QNP and QME) route. Post $15 \mathrm{~min}$ of IN and IV administration of radiolabeled formulations respectively, anesthetized rats were placed in a posterior-anterior position on the imaging board to study the biodistribution. Emitted radiation was captured using a single photoemission computerized tomography gamma camera (Brivo NM 615 gamma camera, GE Healthcare, UK), and scintigraphy images were recorded after 15 min of dosing [19].

\section{Statistical analysis}

All data were reported as mean \pm SD and the differences between the groups were tested using Student's $t$ test (paired $t$ test, two tailed) at the level of $p<0.05$ considered significant and $p>0.05$ non-significant. In case of multiple comparisons, more than two groups were compared using ANOVA (mean of each column is compared with mean of control column using Dunnett's test) and the differences were considered to be significant and highly significant at the level of $p<0.05$ and $p>0.01$ respectively with the help of the GraphPad Prism Version 6.01 software.

\section{Results}

Formulation and characterization of NP and MME

Optimized composition of QNP and physicochemical parameters are depicted in Table 1 . Narrow size distribution $(131.08 \pm 7.45 \mathrm{~nm})$ with PDI of $0.252 \pm 0.064$ was indicative of nano-sized particles. Zeta potential is an electrical charge on the particle surface and acts as a repulsive factor which imparts stability to formulation [17]. Since NP were designed using $\mathrm{CH}$, positive zeta potential value of $34.4 \pm 1.87 \mathrm{mV}$ was attributed to the cationic nature of $\mathrm{CH}$. On the other side, the surfactants (Tween 80 and Labrasol) used in the formulation of ME were non-ionic in nature; therefore, a slight positive or close to neutral zeta potential value of $2.77 \pm 0.51 \mathrm{mV}$ was observed for QME, while QMME showed a positive zeta potential $20.29 \pm 1.23 \mathrm{mV}$ due to presence of an outer surface layer of $\mathrm{CH}$. As per Table 2, oil:S $\mathrm{S}_{\text {mix }}$ :water ratio of $6: 44: 50 \% \mathrm{w} / \mathrm{w}$ resulted in optimized ME formulation, which showed lowest globule size, highest stability, and absence of phase separation upon centrifugation at 5000, 10,000, and $15,000 \mathrm{rpm}$ indicating superior stability. Globule size of QME $(29.75 \pm 0.99$ $\mathrm{nm})$ and QMME $(35.31 \pm 1.71 \mathrm{~nm})$ was comparatively very narrow against QNP $(131.08 \pm 7.45 \mathrm{~nm})$. Morphological analysis by TEM was in line with size distribution by Zetasizer and showed spherical-shaped narrow globules for QME and QMME, while QNP showed coreshell structural morphology (Fig. 1). The pH of QNP, QME, and QMME were within the range of human nasal mucosa, i.e., 4.5 to 6.5 , indicating safe and nonirritant nature of the formulations. Drug loading was > 95\% in case of the ME system, while NP showed poor loading of $64.29 \pm 2.12 \%$. Viscosity for QNP, QME, and QMME were $7.54 \pm 0.22,17.5 \pm 0.69$, and $38.5 \pm 1.26 \mathrm{cP}$ respectively, indicating very low viscosity of QNP and 


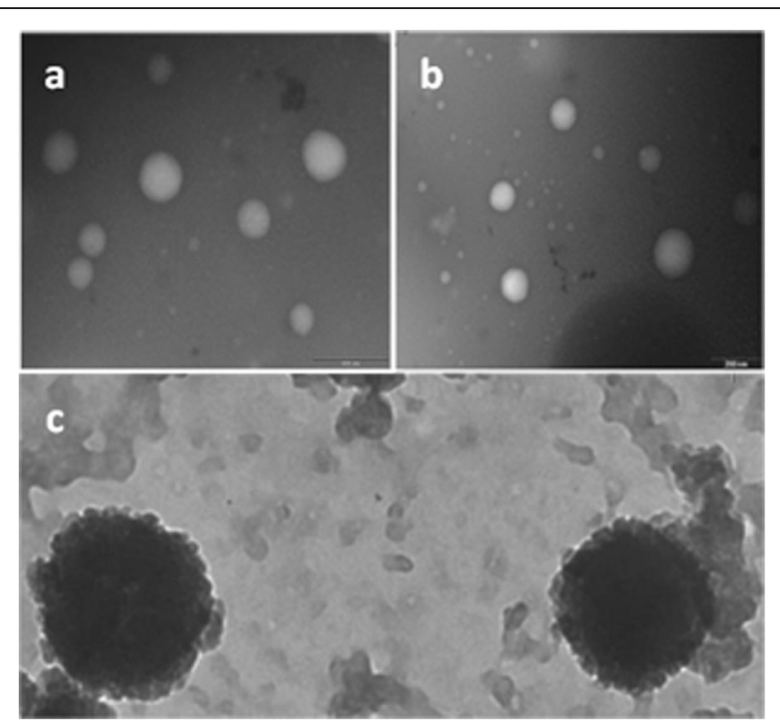

Fig. 1 TEM analysis of quetiapine loaded (a): microemulsion (QME), (b): mucoadhesive microemulsion (QMME) and (c): nanoparticles (QNP)

highest for QMME due to presence of an additional mucoadhesive agent $(0.5 \% \mathrm{w} / \mathrm{v} \mathrm{CH})$.

\section{Nasal diffusion study}

Amount of quetiapine (\%) diffused from QNP, QME, QMME, and DS across nasal mucosa is depicted in Fig. 2. After $12 \mathrm{~h}$, drug diffusion (\%) was in the order of QMME $>$ QNP $>$ QME $>$ DS, i.e., $80.02 \pm 2.03>62.24 \pm$
$1.86>59.38 \pm 3.41>42.93 \pm 3.26$ respectively. Amount of drug diffused was highest from QMME, while QME and QNP showed almost similar release profile.

\section{Pharmacokinetic study}

Quantitative biodistribution study was performed in Sprague Dawley rats, whereby mean brain and plasma distribution profile of quetiapine against different time intervals following IN and IV administration is shown in Fig. 3a and b respectively. Table 3 represents various pharmacokinetic parameters for individual groups. As per Fig. 3a, QMME (IN) showed significantly higher brain distribution at all time points which was followed by QNP (IN) and QME (IN), while the lowest brain concentrations were observed with DS (IN), QME (IV), and QNP (IV) ranging from 60-80 ng/gm. In contrast, the highest plasma concentrations were seen after IV administration of QME and QNP, which were followed by QMME (IN), QNP (IN), and QME (IN) (Fig. 3b). $C_{\max }$ value with QMME (IN) $(243.19 \pm 26.97 \mathrm{ng} / \mathrm{gm})$ was nearly 1.4-folds higher compared to QNP (IN) $(169.27 \pm 20.86 \mathrm{ng} / \mathrm{gm})(p<0.05, n=4)$. Brain/blood ratio results (Fig. 4) also revealed similar findings and supported above stated pharmacokinetic outcomes. At all time points brain/blood ratio of QMME (IN) remained almost 1.2-2.0-folds higher compared to QNP (IN), while with IV dosing it was 3.0-5.0-folds lower at all time points compared to IN dosing thereby indicating higher and prolonged retention of quetiapine from IN formulations, more specifically from QMME at the site

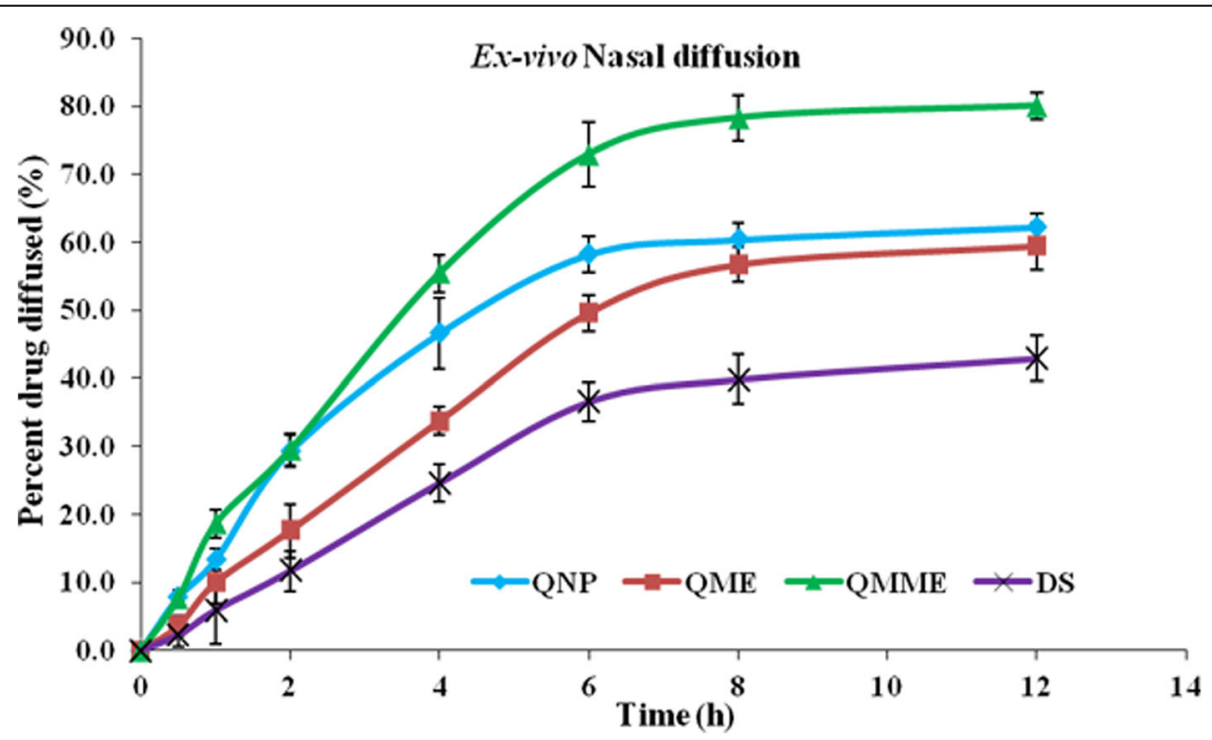

Fig. 2 Comparative ex-vivo nasal diffusion of quetiapine loaded nanoparticles (QNP), microemulsion (QME), mucoadhesive microemulsion (QMME) and drug solution (DS) in phosphate buffer saline (PBS) pH 6.4 using goat nasal mucosa as a dialyzing membrane. (Data represents mean $\pm \mathrm{SD}, n=3)$ 


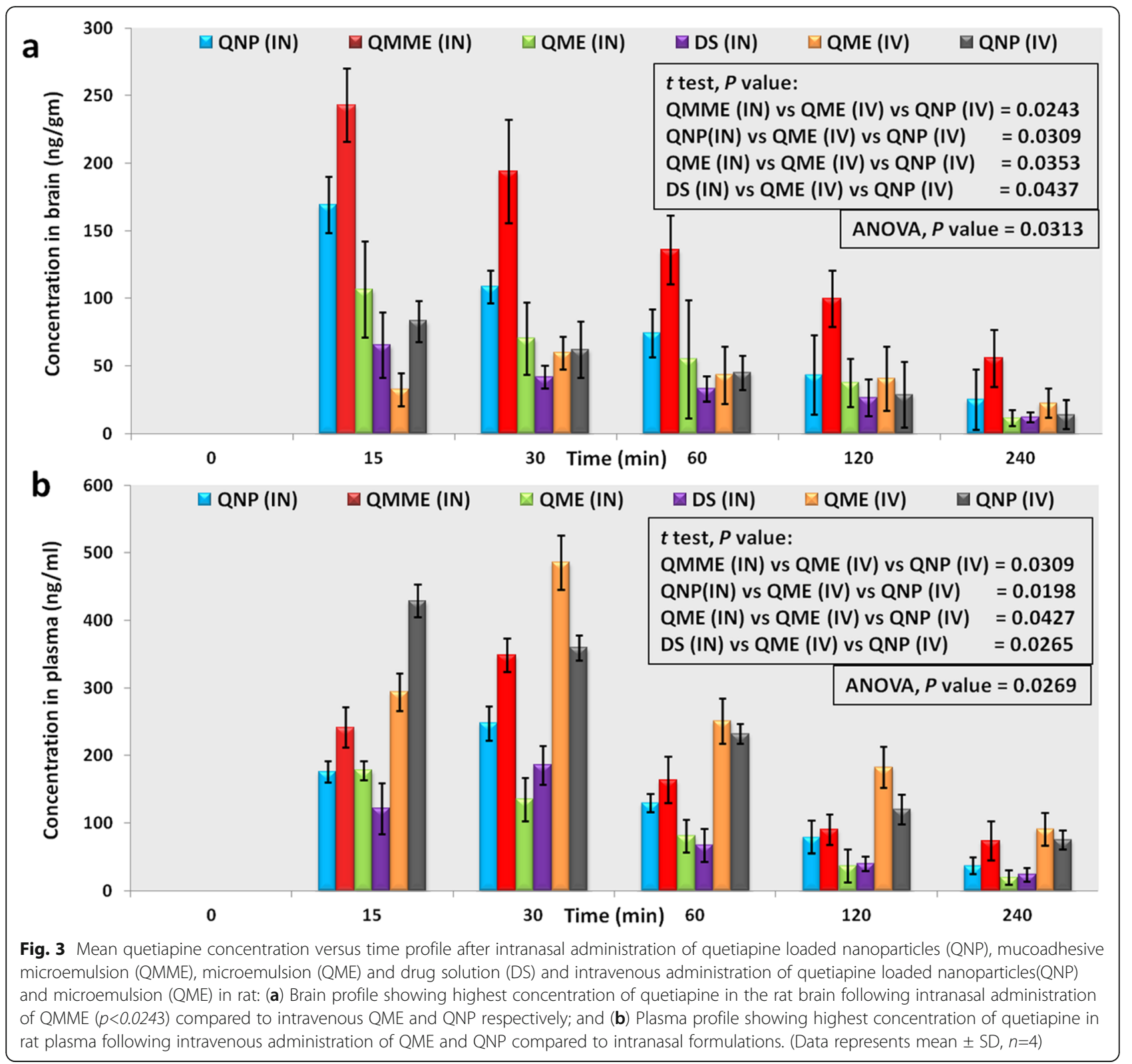

of action. Nearly twofolds, 1.2-folds, and 1.9-folds higher AUC, brain targeting efficiency, and nasal bioavailability was observed respectively for QMME (IN) compared to QNP (IN).

\section{In vivo biodistribution by gamma scintigraphy study}

Gamma scintigraphy study was performed to evaluate qualitative biodistribution following IN (QNP, QMME, QNE, and DS) and IV (QNP and QME) administration of radiolabeled formulations and to support quantitative pharmacokinetic outcomes. Radiolabeling through direct labeling method using reduced ${ }^{99 \mathrm{~m}} \mathrm{Tc}$ showed radiolabeling efficiency for reduced/hydrolyzed $(\mathrm{R} / \mathrm{H}){ }^{99 \mathrm{~m}} \mathrm{Tc}$ and free ${ }^{99 \mathrm{~m}} \mathrm{Tc}$ was within 97 to $98.5 \%$ for all groups. Figure 5 depicts comparative gamma scintigraphy images of different groups post $15 \mathrm{~min}$ of IN and IV administration. Among IN groups highest radioactivity was observed with ${ }^{99 \mathrm{~m}}$ Tc labeled QMME (Fig. $5 \mathrm{~b}$ ) and ${ }^{99 \mathrm{~m}} \mathrm{Tc}$ labeled DS (Fig. 5d) showed the lowest radioactivity, which correlates with the $C_{\max }$ and AUC in the brain. Visually radioactivity was not seen in the brain with QNP (IV) and QME (IV), since the radioactivity counts were very poor, i.e., 264 and 149 respectively. Radioactivity counts with ${ }^{99 \mathrm{~m}} \mathrm{Tc}$ labeled QMME (IN) were 3248, 1.5-times higher compared to counts found with ${ }^{99 \mathrm{~m}} \mathrm{Tc}$ labeled QNP (2116), this data were in line with $C_{\max }$, nasal bioavailability, and brain 
Table 3 Pharmacokinetics, brain targeting efficiency (DTE), direct transport percentage (DTP), and nasal bioavailability (NB) of QNP, QMME, QME, and DS following IN and IV administration (data represents mean \pm SD, $n=4$ )

\begin{tabular}{|c|c|c|c|c|c|c|c|}
\hline \multirow[t]{2}{*}{ Parameters } & \multirow[t]{2}{*}{ Parts } & \multicolumn{6}{|c|}{ Formulation and route of administration } \\
\hline & & QNP (IN) & QMME (IN) & QME (IN) & DS (IN) & QME (IV) & QNP (IV) \\
\hline \multirow[t]{2}{*}{$C_{\max }$} & $P^{*}$ & $248.19 \pm 25.49$ & $348.90 \pm 24.62$ & $178.32 \pm 14.06$ & $185.67 \pm 28.56$ & $485.79 \pm 40.56$ & $429.01 \pm 24.24$ \\
\hline & $B^{* *}$ & $169.27 \pm 20.86$ & $243.19 \pm 26.97$ & $106.67 \pm 35.53$ & $65.44 \pm 24.24$ & $59.73 \pm 12.00$ & $83.21 \pm 15.29$ \\
\hline \multirow[t]{2}{*}{$T_{\max }(\min )$} & $\mathrm{P}$ & 30 & 30 & 15 & 30 & 30 & 15 \\
\hline & B & 15 & 15 & 15 & 15 & 30 & 15 \\
\hline \multirow[t]{2}{*}{$A \cup C_{0-240}$} & $\mathrm{P}^{+}$ & $23458.42 \pm 1672.37$ & $31543.4 \pm 2708.12$ & $13902.74 \pm 2370.79$ & $14123.56 \pm 3267.71$ & $48616.57 \pm 1850.82$ & $40343.62 \pm 1145.12$ \\
\hline & $\mathrm{B}^{++}$ & $13776.15 \pm 2021.24$ & $26493.21 \pm 2762.87$ & $9785.12 \pm 3161.44$ & $6557.42 \pm 726.22$ & $8816.71 \pm 2835.91$ & $8152.12 \pm 1835.11$ \\
\hline \multirow[t]{2}{*}{$T_{1 / 2}(h)$} & $P$ & $1.48 \pm 0.23$ & $1.90 \pm 0.58$ & $1.21 \pm 0.30$ & $1.41 \pm 0.22$ & $1.85 \pm 0.38$ & $1.49 \pm 0.28$ \\
\hline & B & $1.48 \pm 0.12$ & $1.99 \pm 0.56$ & $1.16 \pm 0.32$ & $2.02 \pm 0.78$ & $3.02 \pm 1.17$ & $1.55 \pm 0.17$ \\
\hline DTE (\%) & B & $374.93 \pm 15.02$ & $453.69 \pm 10.17$ & $371.20 \pm 12.02$ & $259.14 \pm 15.35$ & & \\
\hline DTP (\%) & B & $73.33 \pm 4.14$ & $80.51 \pm 6.46$ & $68.66 \pm 6.84$ & $54.08 \pm 5.17$ & & \\
\hline \multirow[t]{2}{*}{ NB (\%) } & $P$ & $58.15 \pm 3.18$ & $59.68 \pm 8.73$ & $32.66 \pm 5.24$ & $31.97 \pm 6.16$ & & \\
\hline & $B$ & $168.98 \pm 9.27$ & $316.67 \pm 19.38$ & $118.65 \pm 14.26$ & $83.15 \pm 9.82$ & & \\
\hline
\end{tabular}

$P$ plasma, $B$ brain

"Plasma $C_{\max }: \mathrm{ng} / \mathrm{ml}$

${ }^{* *}$ Brain $C_{\max }: \mathrm{ng} / \mathrm{gm}$

${ }^{+}$Plasma AUC: $\mathrm{ng} \mathrm{min} \mathrm{ml}^{-1}$

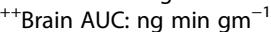

targeting efficiency of QMME, which were 1.4, 1.8, and 1.3-folds higher compared to QNP (IN). As a result of it, direct brain transport percentage with QMME was onefold higher than QNP. ${ }^{99 \mathrm{~m}}$ Tc labeled QME (Fig. 5c) showed 1.7 times lesser radioactivity (1250 Vs 2116 counts) compared to ${ }^{99 \mathrm{~m}} \mathrm{Tc}$ labeled QNP. Radioactivity counts in the brain upon IN administration of ${ }^{99 \mathrm{~m}} \mathrm{Tc}$ labeled DS was only 827 , which was even in the agreement with the lowest $C_{\max }(65.44 \pm 24.24 \mathrm{ng} / \mathrm{gm})$ and poor direct transport amount (nearly $50 \%$ ).

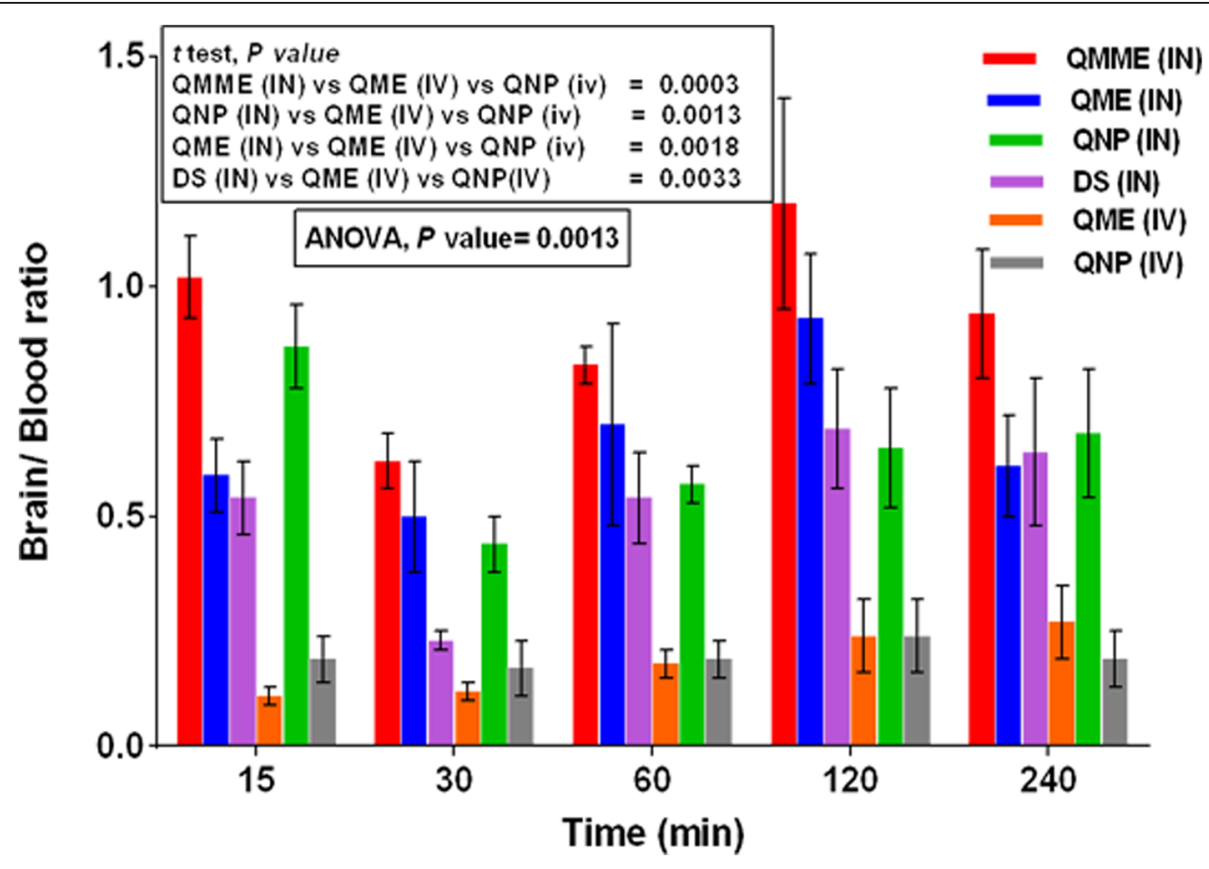

Fig. 4 Brain/blood concentration ratio of intranasally administered quetiapine loaded nanoparticles (QNP), mucoadhesive microemulsion (QMME), microemulsion (QME) and drug solution (DS) and intravenous administration of quetiapine loaded nanoparticles (QNP) and microemulsion (QME) at different time intervals depicting highest ratio with intranasal QMME at all time points, while intranasal QME and QNP showed similar brain/blood ratio but significantly lower than QMME. (Data represents mean $\pm S D, n=4$ ) 

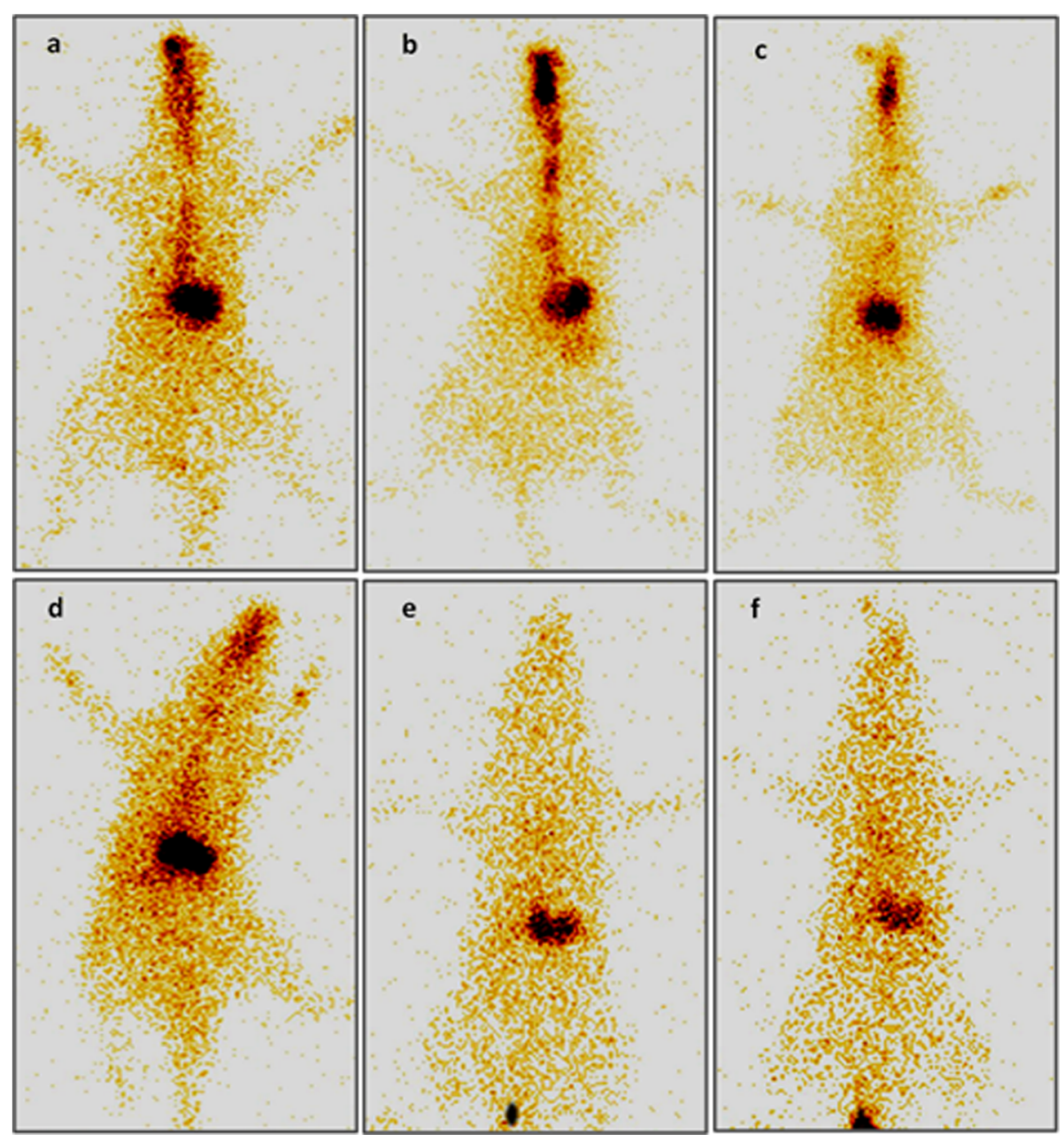

Fig. 5 Gamma scintigraphy images at 15 min post intranasal administration of quetiapine loaded (a) nanoparticles (QNP), (b) mucoadhesive microemulsion (QMME), (c) microemulsion (QME), (d) drug solution (DS) and intravenous administration of quetiapine loaded (e) microemulsion (QME) and (f) nanoparticles (QNP). Among intranasal formulations, QMME represents highest radioactivity in the rat brain which is followed by QNP, QME and DS, while intravenous formulations (e and $\mathbf{f}$ ) did not reveal sufficient radioactivity in the rat brain

\section{Discussion}

Globule size is one of the major factor for nasal delivery of drugs to the brain, since QME and QMME globules were $<100 \mathrm{~nm}$ in size, they will have a larger surface area and can traverse the $\mathrm{BBB}$ more rapidly than QNP [16]. It has been reported by Ahmad et al. that size cutoff plays a crucial role for IN drug delivery. They emphasized on globule size and stated that globules $<100$ $\mathrm{nm}$ in diameter penetrated olfactory bulb and were found in the brain to some extent, while larger particles did not penetrate at all [20]. Highest drug loading in case of the ME system could be attributed to the design of ME containing mixture of oil, surfactant, and cosurfactant into which the maximum amount of quetiapine was solubilized, while poor loading with NP was attributed to poor and $\mathrm{pH}$-dependent aqueous solubility of quetiapine. To achieve higher nasal absorption, the formulation should have prolonged contact with nasal mucosa and hence, viscosity is a very crucial parameter, which directly affects on the residence time of the applied formulations. Optimum viscosity is therefore required to achieve an optimum drug deposition on the nasal mucosa which in turn results in higher nasal absorption. Considering nasal administration of the formulations through device, viscosity is the decisive parameter, which governs the spray characteristics like spray pattern and plume geometry upon actuation. In one finding by Trows et al., it was revealed that when plain water (without polymer) was actuated from the device, very wide plume with fine droplets and highest cone angle $\left(82.1^{\circ}\right)$ was observed. In contrast, a jet-like plume with larger droplets and a lower cone angle $\left(13.2^{\circ}\right)$ was observed, when $5 \%$ polymer solution was actuated from the device [21]. This study suggests that extremely low or too high viscosity results into development of poor spray with uneven droplets which 
may not be suitable for maximum delivery into the upper nasal cavity. However, in our previous report, device characterization evaluation revealed that QMME upon actuation showed cone angle value of $40.85^{\circ}$ and plume length of $10.80 \mathrm{~cm}$, suggesting that QMME in combination with nasal device generated an ideal plume despite its viscosity of $38.5 \mathrm{cP}$, which seems to be optimum for nasal formulations [18]. Higher viscosity of QMME over QME and QNP could be an advantage to overcome MCC and thus it can provide enhanced permeation across nasal mucosa as a result of longer residence time. Contact angle study results supported this statement, whereby QMME had the highest degree of wetting $\left(44^{\circ}\right)$ with nasal mucosa (flat piece of goat nasal mucosa), followed by QME $\left(28^{\circ}\right)$, while QNP did not have sufficient contact with the surface of nasal mucosa due to less viscosity [18].

Poor permeation of quetiapine from DS $(<40 \%)$ was ascribed to the $\mathrm{pH}$-dependent solubility and crystalline nature of quetiapine due to which it could not diffuse across lipophilic nasal mucosa. Higher diffusion with QMME could be ascribed to the presence of cationic $\mathrm{CH}$, which could have interacted electrostatically with the negatively charged sialic acid groups of the nasal mucosa, facilitating superior mucoadhesion, thereby altering the integrity of $\mathrm{TJ}$ present on the epithelial surface of the mucosa. This could either disrupt or modulate the $\mathrm{TJ}$ structure and decrease the strength between adjacent epithelial cells thereby increasing paracellular transport $[22,23]$. This was in agreement with the findings reported by Zerrouk et al., who also stated increased paracellular permeability and TJ modulation effects with $\mathrm{CH}$ [24]. However, it should be noticed that QNP also contained $\mathrm{CH}$ as a polymer, but still showed comparatively poor diffusion against QMME. Probable explanations could be the overall nature of NP and ME system, lipophilic nasal mucosa could have acted as a ratelimiting membrane for the hydrophilic NP, thus hindering and/or lowering the drug diffusion from NP, while lipophilic ME system with unique structure could have overcome this barrier and diffused superiorly [25, 26]. Overall, it can be assumed that $\mathrm{CH}$ alone could not be responsible for enhanced diffusion across nasal mucosa, but versatile composition, mucoadhesion associated with $\mathrm{CH}$, smaller globule size, and lipophilicity together imparted synergistic effect to MME over NP, thus, showed 1.3 times higher nasal diffusion. Additionally, Luciana Lopes explained that, in case of ME, not only small droplet size but large surface area/volume ratio and continuous fluctuating interfaces may increase drug mobility and hence, favor the deeper permeation [27].

Pharmacokinetic data represented that IN administration enhanced concentration of quetiapine in the brain over IV administration, thereby proving two facts: (i) direct connection between the nose and the brain and (ii) lower systemic distribution with IN administration against IV route. As per Table 3, among all IN administered formulations, $C_{\max }$ in the brain was lowest with DS followed by QME < QNP < QMME, suggesting that MCC and residence time significantly affect the drug absorption and permeation across nasal mucosa; hence, nearly 3.8-folds higher brain distribution of quetiapine was seen with QMME compared to DS. Higher $C_{\max }$ value for QMME over QNP could be attributed to smaller globule size and additional permeation enhancing effect of $\mathrm{CH}$ in QMME. Although QME (IN) also had a smaller globule size but still permeation was poor (1.2-folds lower) compared to QNP (IN) and significantly poor (2.3-fold lower) compared to QMME (IN) ( $p$ $<0.05, n=4)$. These results were in line with ex vivo nasal diffusion studies too. Higher nasal bioavailability and brain targeting efficiency for QMME over QNP could be attributed to multiple effects taking place upon IN administration of QMME, viz., (i) mean globule size for QMME was 3.7 times lower than QNP, which could be an advantage for intracellular axonal transport through olfactory neurons [28], allowing direct access across the brain. Electron microscopy studies in 2month-old rabbits have revealed that the average diameter of olfactory axons were about $200 \mathrm{~nm}$ with many axons even less than $100 \mathrm{~nm}$; hence, nanocarriers $<100$ $\mathrm{nm}$ can superiorly transported via olfactory axons to different brain regions [29], (ii) absorption-enhancing effects of $\mathrm{CH}$ on $\mathrm{TJ}$ to facilitate paracellular transport across the epithelial cells of the nasal mucosa. It has been reported that diameter of closed $\mathrm{TJ}$ is in the range of 4-8 $\AA$ and varies to some extent depending upon the leakiness of the epithelium [30]. When certain enhancers are used to widen the $\mathrm{TJ}$ and to promote the paracellular transport, the maximum gap and/or diameter observed was $<20 \mathrm{~nm}$ and hence larger molecules do not pass significantly through the paracellular route [31]. Therefore, smaller globules $(35.31 \pm 1.71 \mathrm{~nm})$ of QMME in the presence of $\mathrm{CH}$ showed higher transport of quetiapine into the rat brain by altering the $\mathrm{TJ}$ integrity compared to QME in spite of its globule size $(29.75 \pm 0.99 \mathrm{~nm})$ and QNP $(131.08 \pm 7.45 \mathrm{~nm})$ and (iii) it has been reported that sometimes NP transport across BBB is diminished due to intracellular accumulation at the surface of endothelial cells, thereby interacting with the reticuloendothelial system (RES) and thus getting cleared rapidly [32]. In contrast, lipidic systems have a higher ability to escape the RES and thus prolonged residence time [16], which could be also one of the reasons for lower concentration of quetiapine with QNP compared to QMME $(p<0.05, n=4)$. In case of QNP, drug was embedded into the $\mathrm{CH}$-TPP matrix formed by crosslinking between them, whereby the transport of quetiapine across nasal 
mucosa depends upon the erosion of matrix and other physiological conditions also. Overall, pharmacokinetic parameters suggested preferential nose to brain transport of quetiapine through QMME (IN) where presence of $\mathrm{CH}$ provided $\mathrm{TJ}$ modulation and mucoadhesion, higher solubility, Pgp inhibition of quetiapine due to ME components, and smaller globules together attributed an ideal condition to overcome biological obstructions and traverse more rapidly and directly into the brain bypassing the $\mathrm{BBB}$.

Lower radioactivity counts with QME (IN) over QNP (IN) could be ascribed to the absence of permeation and mucoadhesion effects in the QME system, because even $C_{\max }$ in the brain with QME (IN) was 1.6 times lower than the QNP (IN). Quetiapine being substrate to Pgp, lowest radioactivity counts and $C_{\max }$ in the brain were obvious after IN administration of DS. As discussed in the pharmacokinetic findings that QNP being a polymeric NP system, shows a higher ability for RES, while lipidic ME system can greatly escape RES. This statement was truly in the agreement with the gamma scintigraphy images. As per Fig. 5a, a relatively significant amount of radiolabeled QNP was deposited in the abdominal region as a result of in vivo nano-bio interactions [33, 34], while in Fig. 5b and c, i.e., with QMME and QME, it was comparatively lower, suggesting longer residence with ME system and rapid clearance with NP formulation. Smaller and/or negligible radioactivity counts found in the brain (visually not seen) after IV administration of QME and QNP could be ascribed to the systemic distribution of quetiapine crossing BBB to a very lesser extent. Overall, qualitative data of gamma scintigraphy study was in correlation with the quantitative pharmacokinetic results and suggested that the brain delivery of Pgp substrate molecule quetiapine through nasal route was significantly higher in the form of MME system compared to NP. In vivo biodistribution and findings of imaging study proved the potential of MME as a choice of delivery system in the brain targeting of therapeutics via non-invasive IN route.

\section{Conclusion}

Present investigation was aimed at evaluating the potential of the nanocarrier system in terms of brain targeting via nasal delivery. IN formulations of quetiapine, viz., polymeric NP, ME, and MME system were developed, whereby physicochemical parameters showed lowest globule size and highest drug loading for ME and MME over NP. Nasal diffusion study revealed enhanced permeation of quetiapine across nasal mucosa from MME compared to NP. In vivo studies showed maximum brain transport of quetiapine through direct olfactory and trigeminal pathway bypassing BBB and highest nasal bioavailability with QMME over QNP, which was further confirmed through gamma scintigraphy study. Outcomes from diffusion and biodistribution studies supported previous findings such as permeation enhancing effects of $\mathrm{CH}$ capable of modulating $\mathrm{TJ}$ and unique lipidic composition of ME providing improved brain targeting. However, these findings do not discourage other nanocarriers, and based on one such comparative finding, it would not be sufficient to comment whether size alone or versatile system of MME together with size is responsible for enhanced olfactory and brain distribution of quetiapine. In the near future, more comparative nose to brain evaluations should be carried out in relevant animal models to explore the potential of non-invasive IN route in combination with ME and MME systems.

\section{Abbreviations}

BBB: Blood-brain barrier; CNS: Central nervous system; CH: Chitosan; DS: Drug solution; IN: Intranasal; IV: Intravenous; MCC: Mucociliary clearance; ME: Microemulsion; MME: Mucoadhesive microemulsion; NP: Nanoparticles; Pgp: P-glycoprotein; Q: Quetiapine; $S_{\text {mix }}$ : Surfactant-cosurfactant mixture; TJ: Tight junction; TPP: Sodium tripoly phosphate; ${ }^{99 \mathrm{~m}} \mathrm{Tc}$ : Technetium-99 m

\section{Acknowledgements}

Authors are grateful to B.V. Patel PERD Centre and Industries commissionerate (Govt. of Gujarat, India) for providing research facilities. The authors would like to acknowledge the head, Department of Pharmacology and Toxicology at B.V. Patel PERD Centre, Ahmedabad for providing animal facilities.

\section{Authors' contributions}

BS and DK are the researchers who carried out the research activities. BS has designed the formulations, performed characterizations, and drafted the manuscript. DK has contributed in the pharmacokinetic experiments. MM as a supervisor guided in writing of the discussion and suggested necessary changes in the drafting of the entire manuscript. All authors read and approved the final manuscript.

\section{Funding}

BS is thankful to Lady Tata Memorial Trust (Bombay, India) for providing the senior research scholar award as financial assistance. MM is thankful to the Department of Science and Technology (Faculty INSPIRE Grant No: IFA-LSBM13, Delhi, India and SERB EMR Grant No: EMR/2016/007966).

Availability of data and materials

All data and materials are available upon request.

\section{Ethics approval and consent to participate}

Animals were provided by the Department of Pharmacology and Toxicology, B.V. Patel PERD Centre, Gujarat, India. Animal experiments and study protocols were approved by the Institutional Animal Ethics Committee of B.V. Patel PERD Centre, Gujarat, India (Registration No: 1661/PO/A/12/ CPCSEA) under CPCSEA, Delhi, India. All institutional and national guidelines for the care and use of laboratory animals were followed. Any animal studies reported in this article were in accordance with the ethical standards of the institution and performed only after obtaining ethical approval from the institutional animal ethics committee.

\section{Consent for publication}

Not applicable.

\section{Competing interests}

The authors report no competing interests.

\section{Author details}

${ }^{1}$ Department of Pharmaceutics, B. V. Patel PERD Centre, Ahmedabad 380054, India. ${ }^{2}$ Department of Pharmaceutical Sciences, College of Pharmacy, University of North Texas Health Science Center, Fort Worth, TX 76107, USA. 
${ }^{3}$ Department of Pharmaceutics, NIPER Ahmedabad, Gandhinagar 382355, India.

Received: 9 October 2020 Accepted: 7 December 2020

Published online: 07 January 2021

\section{References}

1. Zhang SSY (2016) Medical big data: neurological diseases diagnosis through medical data analysis. Data Sci Eng 1:54-64

2. Crowe TP, Greenlee MHW, Kanthasamy AG, Hsu WH (2018) Mechanism of intranasal drug delivery directly to the brain. Life Sci. 195:44-52

3. Patel K, Clifford DB (2014) Bacterial brain abscess. The Neurohospitalist 4: 196-204

4. Illum L (2000) Transport of drugs from the nasal cavity to the central nervous system. Eur J Pharm Sci 11:1-18

5. Balin BJ, Broadwell RD, Salcman M, el-Kalliny M (1986) Avenues for entry of peripherally administered protein to the central nervous system in mouse, rat, and squirrel monkey. J. Comp. Neurol. 251:260-80.

6. Hanson LR, Frey WH (2008) Intranasal delivery bypasses the blood-brain barrier to target therapeutic agents to the central nervous system and treat neurodegenerative disease. BMC Neurosci. 9:S5

7. Illum L (2003) Nasal drug delivery--possibilities, problems and solutions. J Control Release 87:187-198

8. Illum L (2012) Nasal drug delivery - recent developments and future prospects. J Control Release 161:254-263

9. Vyas TK, Babbar AK, Sharma RK, Misra A (2005) Intranasal mucoadhesive microemulsions of zolmitriptan: preliminary studies on brain-targeting. J Drug Target 13:317-324

10. Vyas TK, Babbar AK, Sharma RK, Singh S, Misra A (2006) Intranasal mucoadhesive microemulsions of clonazepam: preliminary studies on brain targeting. J Pharm Sci 95:570-580

11. Reger MA, Watson GS, Frey WH, Baker LD, Cholerton B, Keeling ML, Belongia DA, Fishel MA, Plymate SR, Schellenberg GD, Cherrier MM (2006) Effects of intranasal insulin on cognition in memory-impaired older adults: modulation by APOE genotype. Neurobiol Aging 27:451-458

12. Owen MJ, Sawa A, Mortensen PB (2016) Schizophrenia. Lancet 388:86-97

13. Seeman P (2004) Atypical antipsychotics: mechanism of action. Focus 47: 27-58

14. Soeiro DESMG, Dias W, Missio G, Balanza-Martinez V, Valiengo L, Carvalho AF, Moreno RA (2015) Role of quetiapine beyond its clinical efficacy in bipolar disorder: from neuroprotection to the treatment of psychiatric disorders (Review). Exp Ther Med 9:643-652

15. Brioschi AM, Calderoni S, Zara GP, Priano L, Gasco MR, Mauro A (2009) Solid lipid nanoparticles for brain tumors therapy: state of the art and novel challenges. In: Sharma HS (ed) Progress in brain research 180:193-223. Elsevier.

16. Rajshree L, Shinde ABJ, Devarajan PV (2011) Microemulsions and nanoemulsions for targeted drug delivery to the brain. Current Nanoscience 7:119-133

17. Shah B, Khunt D, Misra M, Padh H (2016) Application of Box-Behnken design for optimization and development of quetiapine fumarate loaded chitosan nanoparticles for brain delivery via intranasal route*. Int J Biol Macromol 89:206-218

18. Shah B, Khunt D, Misra M, Padh H (2016) Non-invasive intranasal delivery of quetiapine fumarate loaded microemulsion for brain targeting: formulation, physicochemical and pharmacokinetic consideration. Eur J Pharm Sci 91: 196-207

19. Shah B, Khunt D, Misra M, Padh H (2018) Formulation and in-vivo pharmacokinetic consideration of intranasal microemulsion and mucoadhesive microemulsion of rivastigmine for brain targeting. Pharm Res 35:8

20. Ahmad E, Feng Y, Qi J, Fan W, Ma Y, He H, Xia F, Dong X, Zhao W, Lu Y, Wu W (2017) Evidence of nose-to-brain delivery of nanoemulsions: cargoes but not vehicles. Nanoscale 9:1174-1183

21. Trows S, Wuchner K, Spycher R, Steckel H (2014) Analytical challenges and regulatory requirements for nasal drug products in Europe and the U.S. Pharmaceutics 6:195-219

22. Yeh TH, Hsu LW, Tseng MT, Lee PL, Sonjae K, Ho YC, Sung HW (2011) Mechanism and consequence of chitosan-mediated reversible epithelial tight junction opening. Biomaterials 32:6164-6173

23. Aderibigbe BA, Naki T (2019) Chitosan-based nanocarriers for nose to brain delivery. Applied sciences 9:2219
24. Zerrouk N, Corti G, Ancillotti S, Maestrelli F, Cirri M, Mura P (2006) Influence of cyclodextrins and chitosan, separately or in combination, on glyburide solubility and permeability. Eur J Pharm Biopharm 62:241-246

25. He CX, He ZG, Gao JQ (2010) Microemulsions as drug delivery systems to improve the solubility and the bioavailability of poorly water-soluble drugs. Expert Opin Drug Deliv 7:445-460

26. Piao HM, Balakrishnan P, Cho HJ, Kim H, Kim YS, Chung SJ, Shim CK, Kim DD (2010) Preparation and evaluation of fexofenadine microemulsion for intranasal delivery. Int J Pharm 395:309-316

27. Lopes LB (2014) Overcoming the cutaneous barrier with microemulsions. Pharmaceutics 6:52-77

28. Gartziandia O, Egusquiaguirre SP, Bianco J, Pedraz JL, Igartua M, Hernandez RM, Préat V, Beloqui A (2016) Nanoparticle transport across in vitro olfactory cell monolayers. Int J Pharm 499:81-89

29. Pardeshi CV, Belgamwar VS (2013) Direct nose to brain drug delivery via integrated nerve pathways bypassing the blood-brain barrier: an excellent platform for brain targeting. Expert Opin Drug Deliv 10:957-972

30. Mistry A, Stolnik S, Illum L (2009) Nanoparticles for direct nose-to-brain delivery of drugs. Int J Pharm 379:146-157

31. Miyamoto M, Natsume H, Satoh I, Ohtake K, Yamaguchi M, Kobayashi D, Sugibayashi K, Morimoto Y (2001) Effect of poly-L-arginine on the nasal absorption of FITC-dextran of different molecular weights and recombinant human granulocyte colony-stimulating factor (rhG-CSF) in rats. Int J Pharm 226:127-138

32. Lu CT, Zhao YZ, Wong HL, Cai J, Peng L, Tian XQ (2014) Current approaches to enhance CNS delivery of drugs across the brain barriers. Int J Nanomed 9:2241-2257

33. Zhang YN, Poon W, Tavares AJ, McGilvray ID, Chan WCW (2016) Nanoparticle-liver interactions: cellular uptake and hepatobiliary elimination. J Control Release 240:332-348

34. Bujie Du MY, Zheng J (2018) Transport and interactions of nanoparticles in the kidneys. Nature Reviews Materials 3:358-374

\section{Publisher's Note}

Springer Nature remains neutral with regard to jurisdictional claims in published maps and institutional affiliations.

\section{Submit your manuscript to a SpringerOpen ${ }^{\circ}$ journal and benefit from:}

- Convenient online submission

- Rigorous peer review

- Open access: articles freely available online

High visibility within the field

- Retaining the copyright to your article

Submit your next manuscript at $>$ springeropen.com 